\title{
Representaciones de apego de niños y niñas con obesidad y la respuesta sensible de sus madres
}

\section{Attachment representation of children with obesity and the sensitive response of their mothers}

\author{
Andrea Tognarelli Guzmán ${ }^{1}$ \\ Universidad Santo Tomás, Santiago, Chile \\ (Rec: septiembre de 2012 - Acep: noviembre de 2012)
}

\begin{abstract}
Resumen
En el presente estudio se establecieron relaciones entre la respuesta materna sensible, el apego y la obesidad infantil. El diseño fue de tipo descriptivo-exploratorio, se utilizó entrevista semiestructurada, el Child Attachment Interview y observación no participante. Los resultados señalan que los niños(as) con obesidad presentan un apego inseguro y que la madre responde con un desacoplamiento frente a las necesidades del hijo; la emisión de respuestas de negación del problema, descuido, invalidación de la experiencia subjetiva y falta de sensibilidad para detectar el hambre y la saciedad del hijo, se relacionan con la obesidad. Se concluye que la intervención en obesidad debe incluir la relación afectiva madre-hijo y se proyecta la posibilidad de prevención del trastorno. Palabras clave: obesidad, apego, respuesta sensible, desacoplamiento y descuido.
\end{abstract}

\begin{abstract}
In the present study relations were established among the mother sensitive response, the attachment and the infantile obesity. The design was of descriptive type - exploratory, a semistructured interview was used, the Child Attachment Interview and observation not participant. The results indicate that the children with obesity present an insecure attachment and the mother responds with a disconnection as opposed to the necessities of the child; the emission of responses of denial of the problem, carelessness, invalidation of the subjective experience and a lack of sensibility to detect the hunger and satiety of the child, it is related with the obesity. One concludes that the intervention in obesity must include the affective relation mother-son and one projects the possibility of prevention of the disorder
\end{abstract}

Keywords: obesity, attachment, sensitive response, disconnection and carelessness.

\footnotetext{
1 Correspondencia a: Estero Poniente 5577, Peñalolén. Tel: 02- 3624781. E-mail: avtognar@uc.cl y atognarellig@investigaciones.cl
} 


\section{Introducción}

La obesidad infantil constituye un problema de salud a nivel mundial y en Chile ha tenido un aumento alarmante en la población (Arteaga, 1996, citado en Hidalgo y Gottlieb, 2001). Los pacientes con obesidad deben su alto peso a su conducta de alimentación y su baja actividad diaria. Una parte importante de estas conductas se forma en la interacción con la madre. El apego considera la relación afectiva madre-hijo y permite comprender y predecir el funcionamiento psicológico (Bowlby, 1980; Crittenden, 1988). La madre en la relación de apego es sensible a los requerimientos del hijo, detecta sus necesidades y facilita su respuesta, lo que le permite al niño adaptarse e integrar su experiencia; esta capacidad organizadora de la madre puede presentar deficiencias que se asocian a problemas de salud mental (Barudy, 2005; Bowlby, 1969; Mahoney, 2002). Actualmente se desconoce cómo se relacionan la respuesta materna sensible, el apego y la obesidad de niños y niñas en etapa escolar; es por esto que el objetivo de este estudio fue describir y relacionar el apego, la respuesta materna sensible y las representaciones maternas en torno a la obesidad. La investigación pretende ser un aporte al abordaje terapéutico de la obesidad desde la relación afectiva madre-hijo, ya que hasta ahora la orientación psicológica se ha enfocado en los hábitos de alimentación y la imagen corporal (Buceta, 1996; Fernández, 1994; Miller, 1980); este enfoque de hábitos de alimentación ha tenido muy bajos resultados (Barja, Nuñez, Ibarra, Velardia, Urrejola, Urrejola \& Hodgson, 2001).

\section{Antecedentes teóricos y empíricos}

La obesidad infantil ha tenido un aumento en la población chilena, en niños en etapa escolar alcanzó a un 16,9\% en 2003 (Olivares, Zacarías y Lera, 2005). La importancia de entender la conducta de alimentación desde la relación del niño con su madre puede verse apoyada en el hecho que la obesidad simple, debido a la conducta, suma más del $99 \%$ de los casos. La obesidad simple hiperplásica, que tiene un peor pronóstico, se determina en la infancia, etapa del desarrollo humano en la cual es fundamental el cuidado materno (Carmena, 1985, citado en Belloch, 1995).

Desde la perspectiva analítica se ha abordado la relación del hijo obeso y su madre; Bruch (1973, citado en Plozza y Pöldinger, 1986) señala que los progenitores pueden desencadenar obesidad psicógena en sus hijos en tanto responden a cualquier necesidad que exterioriza el niño con una oferta nutricional. Desde otra mirada, el enfoque cognitivo también aporta datos a la influencia de la madre de niños obesos, específicamente sobre la percepción del peso e imagen corporal de sus hijos. De manera particular, la ingesta excesiva de alimentos de parte del obeso se produce por un déficit en la percepción de hambre y saciedad internos del sujeto (Shachter \& Rodin, 1974, citado en Belloch, 1995). Se ha determinado que la distorsión de la percepción del peso real que hacen los obesos (Fernández, 1994; Raich, Torras y Mora, 1997) también la hacen las madres hacia el peso real de sus hijos (Díaz, 2000; Kain, Olivares, Castillo y Vio, 2001), por lo que es posible pensar que la percepción de la madre sobre el hijo influye en las dificultades de autopercepción del niño con obesidad. El marco de la presente investigación se sustenta en que la madre organiza la experiencia del hijo, por ello se exploró cómo se dificulta esta función en torno a la comida.

La teoría del apego aborda como un comportamiento la relación afectiva madre-hijo y considera la respuesta sensible de la madre o figura de apego como un organizador psíquico del hijo. Las conductas de apego se organizan en torno a representaciones mentales de la relación con una persona específica (Marrone, 2002) y hacen posible la organización de la experiencia subjetiva. Según Ainsworth (1978), es posible identificar, de acuerdo a la conducta del niño hacia su madre, tipos de apego seguro e inseguro, de los que se definen las pautas de apego seguro, inseguro-evitativo e inseguroambivalente (Sroufe, 2000). Se ha demostrado que las representaciones de apego se transmiten transgeneracionalmente (Fonagy, Target y Steele, 1998; Guidano, 1987; Main, Kaplan \& Cassidy, 1985). Los niños con un apego inseguro-evitativo (Marrone, 2002) presentan una inhibición de lo afectivo que les permite reducir el rechazo materno y su propia rabia frente a este rechazo (Crittenden, 1995). Manifiestan una representación de los otros como no disponibles y se representan a sí mismos como fuertes, les cuesta controlar su rabia y exhiben dificultades para afrontar situaciones conflictivas o de frustración (Barudy y Dantagnan, 2005). Los niños con apego inseguro ansioso-ambivalente desarrollan sentimientos de ambivalencia hacia sus cuidadores, quienes han reactivado y luego frustrado las conductas de apego, con muestras de rechazo parcial e intermitente. Los niños incrementan su vulnerabilidad para mantener la proximidad con sus figuras de apego, presentan falta de estabilidad emocional, irritabilidad y pérdida fácil del control. Prefieren la fusión relacional 
en vez de la autonomía (Marrone, 2002; Barudy y Dantagnan, 2005). Los tipos de apego, según las narrativas descritas a partir del instrumento Child Attachment Interview utilizado como base para este estudio (Target, Fonagy \& Shmueli-Goetz, 2003), señalan que en cuanto al estilo de apego inseguro, el patrón inseguro-evitativo aparece con una sub-activación del apego y una sobre regulación emocional. En el inseguro-ambivalente, la estrategia predominante del niño es quedarse preocupado con las figuras de apego y/o en temas repetitivos con respecto a los padres (Lecannelier, 2002).

En cuanto a la obesidad y su relación con el apego, se ha determinado que el apego inseguro se asocia a la organización de significado personal de los Desórdenes Alimentarios Psicógenos (DAP) de tipo obesa. En este tipo de organización el cuidador produce en el niño una experiencia discrepante, generando una confusión del sentido de sí mismo en el niño y una excesiva vinculación hacia el cuidador. Al no poder establecer regularidades claras en torno a sí mismo, el niño no logra reconocer bien sus propias necesidades ni estados internos, teniendo como resultado una percepción de la mayoría de sus impulsos y emociones de un modo rudimentario e incierto, a excepción de la primitiva sensación corporal conectada al hambre y la motilidad (Guidano, 1987). Por otro lado, en cuanto a la sensibilidad materna, se ha observado en pacientes obesos, la deficiencia materna en captar y dar una adecuada solución a sus necesidades (Bustos, Fantuzzi, Greibe y Steiner, s.f.).

La respuesta sensible de la madre se refiere a un organizador psíquico que durante la infancia considera detectar las señales del infante, interpretarlas de manera adecuada y responder de un modo correcto y rápido (Ainsworth, 1974, en Carretero, 1983). En la niñez se expresa en ver al niño con su propia individualidad y necesidades, separado de los demás (Bowlby, 1998). En cuanto a la relación de la respuesta materna sensible con el apego, se ha determinado que si las madres son sensitivas generan vínculos seguros, si son inconsistentes generan vínculos ambivalentes y si interfieren o rechazan generan vínculos evitativos. La falta de sensibilidad en la respuesta materna aparece cuando el cuidador fracasa en leer los estados mentales del bebé o cuando fracasa en apoyarlo en el logro de sus deseos o estados positivos (Marrone, 2002). Las madres de niños con apego inseguro-ambivalente fallan en la disponibilidad emocional y en la sincronía para satisfacer las necesidades de sus hijos, haciendo que los cuidados hacia el hijo sean inconsistentes e impredecibles; el cuidado parental tiende a la negligencia, física y emocional. En cuanto a las madres de niños con apego inseguro evitativo, se comportan de un modo maternalmente irresponsable, con un sentimiento de rechazo al hijo, son oposicionistas a los deseos de sus hijos y expresan aversión al contacto corporal cercano (Ainsworth \& Tracy, 1981; Bowlby, 1997; Barudy y Dantagnan, 2005). A nivel general, las respuestas carentes de sensibilidad de las madres incluyen las comunicaciones patogénicas, tales como: la invalidación de la petición infantil de apoyo y comprensión, negación de la percepción del niño de ciertos eventos familiares, invalidación de la experiencia subjetiva del niño, intrusividad y lectura de la mente y reacciones exageradas. En cuanto a patrones de conducta y actitudes carentes de sensibilidad se encuentra: falta de habilidad para respetar la iniciativa del niño, rechazo, descuido, teniendo este último como factores principales la falta de apoyo emocional y la falta de protección y supervisión adecuadas; falta de habilidad para jugar juntos e inversión de roles, esta última refiere que quien cuida fundamentalmente al otro en la díada padre-hijo es el hijo (Marrone, 2002).

En cuanto a una posible explicación de las conductas carentes de sensibilidad hacia los hijos se encuentra la fuerte tendencia autoconfirmatoria que puede tener un hijo para los padres. Esto aparece evidente en la inversión de la relación padre/hijo, en la cual los padres esperan que sus hijos los cuiden y reparen los dolores de su vida. Las madres de niños con apego inseguro ambivalente esperan satisfacer sus propias necesidades afectivas con los hijos, por lo que cuando estas necesidades se ven frustradas, la maternidad les complica y agobia, disminuyendo los cuidados del hijo, pudiendo paralizarse frente a sus demandas y funcionar negligentemente (Barudy y Dantagnan, 2005).

\section{Metodología}

El presente estudio se enmarcó en el paradigma interpretativo de investigación en ciencias sociales (Guba, 1990). La metodología utilizada fue cualitativa, la que permite trabajar desde los sujetos (Taylor y Bogdan, 1992) en base a conceptos y de modo inductivo establecer relaciones científicas. El tipo de estudio es descriptivo-exploratorio y el análisis se guió por la escuela metodológica de la Grounded Theory (Glaser \& Strauss, 1967, citado en Krause, 1995).

\section{Participantes}

La población corresponde a niños(as) con obesidad en etapa escolar y sus madres, con un nivel 
socioeconómico medio y bajo y que se atienden en consultorios de salud nutricional. La muestra se completó sucesivamente hasta alcanzar 8 díadas madre e hijo(a), es decir, 16 participantes. Los criterios de selección de la muestra fueron: madres con edad mínima de 32 años, hijos(as) con diagnóstico de obesidad en edades de 7 a 12 años, cursar período de inicio del tratamiento para obesidad infantil, ser atendidos en el consultorio de la Facultad de Medicina de la Pontificia Universidad Católica de Chile o en el Centro Integral de la Mal Nutrición de La Serena.

\section{Procedimientos}

a) Contacto con los participantes. Se inició con el acceso a los libros de registro de atención de los centros de atención, en los que era posible conocer la fecha de inicio del tratamiento y la edad del niño(a). Luego eran contactadas vía telefónica las madres, a quienes se les explicaba la naturaleza del estudio.

b) Recolección de información. Para evaluar el apego, se utilizó la primera versión del Child Attachment Interview (Target, et. al., 2003), que contiene preguntas que activan el sistema representacional de apego del niño sin estresarlo, éstas indagan eventos de apego recientes y el modo como ha representado la relación con ambos padres; considera la coherencia y cooperación en el diálogo.

Las categorías para una clasificación de apego inseguro son: restricción en apertura emocional, desbalance de referencias positivas y negativas, escaso uso de ejemplos, baja resolución de conflictos y coherencia total, presencia de idealización, rechazo del apego y rabia preocupada. Por último, se complementó con elementos comunes de las entrevistas a niños(as) en edad escolar y enfatizando los elementos episódicos de las respuestas (Flick, 2002; Martorell y González, 1997). Para la formulación de la entrevista de la respuesta materna sensible se diseñaron preguntas con componentes episódicos en base a situaciones donde se estresa la respuesta materna, en las áreas de exploración del hijo(a), el apoyo, la contención afectiva y la alimentación, así como preguntas que abordaron las representaciones en torno a la obesidad del hijo(a). La técnica de recogida de datos visuales fue la observación no participante y directa, videando el curso de la entrevista, fue sistemática, selectiva y en un entorno privado (Flick, 2002; Pérez Serrano, 1992). Para su codificación se utilizó como base el inventario de conductas no verbales de Martorell y González (1997) y parte del sistema Facial Affect Scoring Techniche (FAST) de
Ekman, Friesen \& Tomkins (1971, citado en Knapp, 1992). Las conductas no verbales consideradas en la observación se agruparon en kinésicas (movimientos corporales como gestos, contacto ocular, postura y expresión facial) y paralingüísticas (nivel de voz y fluidez del habla) con sus respectivos posibles efectos o significados (Martorell y González, 1997). En cuanto a la expresión facial de emociones, se incorporaron los códigos desarrollados por Eckman (1971, citado en Knapp, 1992) en el sistema FAST para las emociones de sorpresa, miedo, cólera, disgusto, felicidad y tristeza.

La aplicación de las entrevistas se efectuó en un espacio privado, sin interrupciones; las entrevistas fueron grabadas en video y audio. Una vez recepcionados los participantes, se les explicaba la naturaleza del estudio, qué se necesitaría de ellos y el resguardo a la identidad y confidencialidad, acompañado del consentimiento informado expreso y escrito (Colegio de Psicólogos, 1999). Luego se aplicaban por separado las entrevistas, primero al niño(a) y después a la madre.

c) Análisis. Durante la recolección de información, se realizó un análisis de contenido, correspondiendo al proceso de codificación abierta; luego de aplicar cada entrevista, éstas eran transcritas textualmente, codificadas e incorporadas sucesivamente a redes descriptivas (Briones, 1982), para las madres y los(as) niños(as) respectivamente; el proceso de codificación fue revisado hasta alcanzar una estabilidad suficiente. Hubo dos codificadores para los casos de los niños(as) de modo de triangular la codificación verbal y observacional del apego, se conservaron teóricamente las categorías de apego del CAI y en base a ellas se tipificó el apego como inseguro o seguro para cada caso. Los datos visuales se analizaron caso a caso triangulando las categorías de apertura emocional, idealización, subestimación y rechazo, coherencia, rabia preocupada y tristeza. Después de la recolección de información se revisaron los conceptos y las posibles hipótesis, para establecer relaciones posibles y suficientemente fundamentadas en los datos. Esto dio inicio al proceso de codificación axial (Krause, 1992). Posteriormente se realizó el análisis selectivo, obteniendo de modo preliminar una teoría desde la base.

La credibilidad de este estudio se sustentó en la observación y triangulación. Se utilizó la triangulación de técnicas de observación y entrevista, por un lado, y la comparación de los datos obtenidos de la madre y el niño, por otro. La consistencia del estudio se fundamentó en desarrollar una pista de revisión en la cual se describen detalladamente el proceso de recogida, el análisis y la interpretación de los datos. 
La confirmabilidad del estudio se fundamentó en: a) recogida de datos lo más concretamente posible, transcripciones textuales y citas directas; b) revisión de hallazgos inter-investigadores; c) recogida mecánica de datos en grabaciones de audio y video (Flick, 2002; Pérez Serrano, 1992). La transferibilidad de los resultados de la investigación a otras situaciones se limita a realizar inferencias a casos muy similares.

\section{Resultados}

\section{Resultados descriptivos}

\section{a) Significados en torno a la maternidad}

En la historia del embarazo, las madres refieren antecedentes de obesidad, riesgo de pérdidas o pérdidas de hijos antes de nacer. A nivel emocional es relevante el significado del hijo para la madre como el que llena el vacío o el que quita su soledad o tristeza. Siguiendo con el nacimiento, es relevante el estado del hijo en cuanto al peso; observándose casos con bajo peso al nacer, lo contradice la idea de que el niño obeso nace gordo y permite pensar que al encontrarse con un hijo de bajo peso, la madre reacciona con más ansiedad de lo normal para que el niño engorde.

\section{b) Significados en torno a la obesidad}

Se encontró en las madres una valoración positiva de la obesidad que asocia la gordura con salud y belleza en el infante. En la actualidad definen la obesidad como un problema; empero, niegan o no ven este problema en sus hijos, tampoco recuerdan el peso de sus hijos o lo confunden. Esto hace que el proceso de identificación de la obesidad del hijo se haga de modo tardío, al menos tres años después de la aparición de una obesidad evidente. En cuanto al origen de la obesidad reconocen la genética, problemas en las pautas de alimentación y el anhelo de que la obesidad se deba a un problema aún no detectado. Lo anterior muestra que contrario a lo hallado en los estudios con pacientes obesos ${ }^{2}$, ellas atribuyen las causas a factores innatos o causas médicas desconocidas en conjunto con los hábitos de alimentación. Se piensa que esta "distribución de responsabilidad" en relación a la obesidad impide una mayor valoración de la importancia de los hábitos de

\footnotetext{
2 Carmena, 1985, citado en Belloch, 1995, refiere sólo el 1\% de los
} casos a causas genéticas o endocrinas. alimentación y estilos de vida para esta enfermedad, pudiendo mantener en el tiempo hábitos poco saludables. Por otro lado, se desconoce hasta qué punto la importancia señalada a los hábitos de alimentación se deba a un efecto del tratamiento que cursan sus hijos. Desde la respuesta materna, la negación y minimización de un problema tan evidente y de larga data, así como la distribución de responsabilidad es comprensible por el uso de estrategias negligentes, que incluyen el traslado de la responsabilidad a cualquier otro, y enfrentar los problemas rechazándolos o negándolos (Crittenden, 1988).

Una consideración en cuanto al posible rol de la madre en la obesidad del hijo(a) se encuentra en las rutinas de alimentación que la madre establece. En la etapa de la niñez la madre entrega comidas de alto contenido calórico para gratificar y consentir al hijo(a) así como por su propia comodidad. Dentro de las rutinas de alimentación, es posible analizar aspectos de la relación madre-hijo(a) en torno a la comida, ya que el(la) hijo(a) presenta problemas para comer desde la infancia hasta ahora, ya que rechaza las comidas que la madre le entrega y es el propio niño quien determina comer en exceso alimentos altamente calóricos. La madre no logra enfrentar la necesidad de su hijo de ser guiado. Lo anterior en términos de apego señala a una madre de un niño con apego inseguro-ambivalente, es decir, que espera satisfacer sus propias necesidades afectivas con su hijo y que frente al fracaso se paraliza, funcionando negligentemente (Ainsworth \& Tracy, 1981; Barudy y Dantagnan, 2005).

\section{c) La dificultad de la respuesta sensible en madres de niños con obesidad}

1) Descuido. Se evidenció como falta de apoyo emocional cuando el niño lo necesita, como por ejemplo cuando la madre se asusta excesivamente frente a las caídas del (la) hijo(a). Otras dimensiones del descuido son la falta de protección y supervisión adecuadas que se evidencia en el no poder mantener los horarios de comida. Es posible pensar que las situaciones de peligro del niño la cuestionan y superan emocionalmente, no pudiendo llegar a responder, estas son las características de un cuidador que facilitan un apego ansioso. En cuanto a la obesidad, el descuido de los horarios sugiere la presencia de estrategias negligentes que facilitan el mantenimiento de la obesidad.

Falta de habilidad para jugar juntos: se expresa en no participar en el juego del hijo(a) o en la imposibilidad de participar en un juego simbólico juntos, esto muestra a una madre poco disponible mentalmente para su hijo, que le cuesta empatizar con su estado subjetivo. 
2) Problemas con el control y contención sobre la conducta del hijo. Existe una percepción general de dificultad para controlar la ingesta de alimentos del hijo, así como una sensación de angustia frente a la ansiedad del hijo por comer. Por otro lado, la madre facilita la trasgresión de la dieta del hijo. Los resultados sugieren la incapacidad de contener los estados emocionales del hijo por sobre los propios, así como la cadena de frustración en el rol-déficit en la respuesta materna; la trasgresión de la dieta que la madre realiza apunta a un comportamiento intrusivo y contradictorio, que facilita la ansiedad y la ambivalencia en el niño.

3) Inversión de roles. Aparece en conductas de cuidado, protección y comprensión que realiza el niño hacia los padres cuando son ellos los que deberían emitir esa respuesta al hijo. Este es un indicador de dificultades en la respuesta materna sensible e inseguridad del apego.

4) Dificultad para detectar hambre y saciedad del hijo. Se observó que la madre no considera las claves verbales y no verbales del niño para detectar cuando tiene hambre o está satisfecho. La madre puede no haber notado nunca al hijo sin hambre o haberle dado comida sin saber si tenía hambre. Por otro lado, puede determinar si el hijo está satisfecho sólo siguiendo horarios o cantidades. Se piensa que esta restricción de la madre para reconocer el hambre y saciedad del hijo accediendo a sus estados internos es traspasada como repertorio de conductas al hijo, de modo que éste tampoco puede reconocer su propia hambre y saciedad.

5) Dificultad para respetar la autonomía del hijo. Se manifestó cuando la madre obliga a comer al hijo, por ejemplo no permitiendo que deje comida en el plato, haciéndolo comer aunque no lo desee o insistiendo en darle comida aunque el niño señale no querer más. Los datos anteriores nos muestran la sobreprotección materna que impide la propia regulación de la alimentación en el hijo, ya que invalida el reconocimiento del propio estado biológico. En términos de apego es una madre que facilita un apego inseguro-ambivalente.

6) Reacciones exageradas, amenazas y otras comunicaciones. Las reacciones exageradas en torno a la exploración del niño incluyen no dejar que los hijos salgan de la casa a jugar y la excesiva angustia de los padres cuando dejan que se quede fuera. En cuanto a la obesidad, las comunicaciones patógenas en el transcurso del tratamiento intentan controlar la conducta alimentaria del hijo(a); incluyen comentarios que cuestionan los deseos de adelgazar del hijo(a), las críticas improductivas, ridiculizar al hijo(a) para que no coma e imponer malos tratos, retándolos(as) por ser gordos(as) o amenazando con los consecuentes daños físicos a causa de la obesidad. En términos de apego y respuesta sensible, es posible pensar en las respuestas exageradas como una expresión de sobreprotección que junto a las amenazas y otras comunicaciones patógenas favorecen la inseguridad del apego; se piensa además que estas comunicaciones influyen en una respuesta oposicionista de parte del hijo, exacerbando la ingesta en vez de reducirla.

7) Invalidación de la experiencia subjetiva. Se manifestó por comunicaciones que señalan redefiniciones sobre la percepción real del niño, ya sea frente al conflicto de los padres como en la alimentación. En esta última se puede ver en acciones para obligar al hijo a comer durante el tratamiento, lo que implica una dificultad de la madre para respetar la regulación de la saciedad que el niño está tratando de entrenar. Estas comunicaciones se relacionan con inseguridad del apego, ya que el niño debe depender constantemente de la subjetividad de la madre para definir sus propios estados, limitando su propia capacidad de ordenar mentalmente la experiencia. Por otro lado, la invalidación sobre el intento de regulación con la comida señala un mensaje contradictorio al hijo.

\section{d) Representaciones de apego de los (las) niños(as)}

1) Imagen, conflictos y la subestimación de las figuras de apego. Se observó una dificultad en la descripción de la interacción con la madre ya que: aparecen cualidades positivas en desbalance con las negativas, las interacciones positivas aparecen poco ilustradas con ejemplos y el relato aparece poco coherente y poco cooperador. La categoría idealización de la figura de apego materna muestra que las afirmaciones positivas sustentadas consistentemente con ejemplos son escasas. En cuanto a la subestimación del apego, aparece frente a situaciones de lesiones, caídas o enfermedades. En la resolución de conflicto se observa la ausencia de solicitud de ayuda del hijo(a) a los padres. No obstante, se observa una dificultad para afrontar conflictos por sí solo, dejando pasar los conflictos, deseando que otro responda por él o dejando que la madre intervenga.

2) Restricción en la apertura emocional y la expresión de rabia. Se observó dificultades de expresar asertivamente el enojo, teniendo como respuesta común encerrarse a solas, no hablar, acostarse o irse. La restricción de la apertura emocional se pudo apreciar en olvidos reiterados de situaciones conflictivas con los padres y la inexpresión frente a situaciones críticas. La dificultad en la regulación de emociones se refleja en torno a la ira, mostrando una expresión incontenible 
de rabia hacia la madre. Lo anterior da muestras de la presencia de rabia preocupada ${ }^{3}$, indicador del patrón de apego inseguro ambivalente; por otro lado, la evitación de la expresión de emociones es propia del apego inseguro evitativo.

3) Tipos y patrones de apego. Siguiendo las categorías del CAI es posible determinar que en siete de los(as) niños(as) se observó una clasificación de apego inseguro. En cuanto a los patrones de apego, el análisis de los resultados ubica un caso como inseguro evitativo y los otros seis como inseguro ambivalente; en el primero aparece la inhibición de lo afectivo (Crittenden, 1995), la representación de los otros como no disponibles y a sí mismos como fuertes; la dificultad para controlar su rabia y afrontar situaciones conflictivas o de frustración, la idealización de los padres o la descripción de sus limitaciones sin ninguna afección emocional (Barudy y Dantagnan, 2005). En los seis restantes, de tipo inseguro-ambivalente, se encontró el quedarse preocupado con las figuras de apego y/o en temas repetitivos, la preocupación de daño frente a la lejanía de la madre (Lecannelier, 2002); los reclamos de atención y afecto, la manifestación y amplificación de vulnerabilidad (Marrone, 2002; Barudy y Dantagnan, 2005). En todos los casos aparece la representación de los otros como no disponibles, expresado en la respuesta de nunca solicitar ayuda frente a los conflictos ${ }^{4}$.

\section{Resultados analítico relacionales}

Los siguientes resultados muestran tres fenómenos que permiten establecer relaciones entre respuesta materna sensible, representaciones de apego y la obesidad.

1) La invalidación de la experiencia subjetiva. Alude a la actitud de la madre de ignorar la experiencia interna del hijo(a). Tiene como causas la sobreprotección. Se expresa en la dificultad para jugar juntos o compartir el mundo de imaginación del niño, cuando la madre niega la percepción de sus conflictos conyugales, así como cuando niega la petición de comprensión, afecto y apoyo que el niño le hace, respondiendo a sus necesidades con reacciones exageradas, amenazas, maltrato y comunicaciones patógenas. Las consecuencias en el niño(a) son el apego inseguro ya que nunca sabe cuándo su madre estará disponible frente a su experiencia subjetiva, la inversión de roles o hacerse cargo de la madre en aquello que se mostró incapaz de

\footnotetext{
3 El niño expresa su rabia o quejas incontenibles o exageradas hacia sus figuras de apego.

4 Véase en figura 1: el conflicto y subestimación de las figuras de apego.
}

hacer, la rabia preocupada, la idealización materna y la subestimación de la figura de apego.

2) La falta de sensibilidad para detectar el hambre y la saciedad. Hace referencia a la dificultad de las madres de detectar el hambre y la saciedad del hijo(a), no fijándose en claves corporales, no preguntándole al hijo(a), no viéndolo satisfecho o sin ganas de comer. Incluye el guiarse exclusivamente según indicaciones externas al niño(a) para identificar el hambre y la saciedad. Tiene como causas la invalidación de la experiencia subjetiva en torno a las sensaciones corporales que el niño tenga; otras causas son las reacciones exageradas respecto a la alimentación, como tornarse ansiosa si el niño no come aunque esté obeso, la intrusividad y lectura de mente en tanto cree adivinar los estados del hijo y la sobreprotección materna en tanto no lo deja regular sus propias funciones. El contexto de este fenómeno es que las madres tienen una valoración positiva del hijo gordo, así como la evaluación de la función materna principalmente como función de alimentación, la falta de comprensión de la crianza como sintonía con el niño(a) y la percepción de incapacidad del niño de regular sus propias funciones. Las acciones de la madre incluyen dar comida al hijo(a) sin saber si tiene hambre, maltrato asociado a la comida, comunicaciones patógenas para controlar la ingesta, obligar al niño a comer aunque esté en tratamiento para bajar de peso y la entrega de refuerzos con alimentación.

Las consecuencias son que el hijo(a) presenta problemas para comer ya que rechaza las comidas que la madre le entrega.

3) El descuido en la alimentación. Alude a la falta de protección y supervisión adecuadas en la alimentación del hijo(a), esto se observa en la dificultad para mantener horarios de alimentación, en la falta de control de la ingesta y en la inducción a la trasgresión de la dieta. Una de las causas del descuido en la alimentación es la inversión de roles, ya que la madre es quien facilita excesos e induce a romper la dieta y no muestra autoridad suficiente.

Otra de las causas es la negación del problema. Se piensa que el hijo genera una compensación al estado emocional de la madre, por los significados que "llenan el vacío de la madre", lo que le impide aceptar concientemente el problema de su hijo.

De manera global influye en el descuido el que las madres dispongan de poco tiempo para cocinar, que abuelas u otros familiares trasgredan la dieta y que los padres se encuentren en conflicto. A nivel particular influye la percepción de falta de control de la madre sobre el hijo(a), el padre ausente y con dificultad para 
poner límites. Las acciones que realiza la madre cuando descuida la alimentación son entregar comida sin restricciones, la falta de límites y horarios, entregar alimentación de alto contenido calórico, compensar con comida y sobre-ofrecimiento de comidas. Las consecuencias del descuido en la alimentación son las dificultades en la obediencia al tratamiento, comer alimentos poco sanos y en exceso, y aumentar de peso sostenidamente.

\section{Resultados selectivos}

La respuesta sensible de las madres de niños(as) con obesidad y las representaciones de apego de los(as) niños(as) se encuentran influidos por un fenómeno central denominado desacoplamiento materno del estado mental del(a) hijo(a), esto es, la madre no logra sintonizarse completamente con las necesidades del hijo(a). Este desacoplamiento está influido por el significado emocional del hijo que llena el vacío de la madre y por la sobreprotección. El desacoplamiento influye en la invalidación de la experiencia subjetiva, en la inversión de roles y en la falta de habilidad para respetar la iniciativa y autonomía del hijo(a). Esta última influye, por un lado, en que la madre tenga reacciones exageradas frente a las necesidades de exploración del hijo(a) $y$, por otro, a que utilice comunicaciones patógenas para controlar la exploración y la disciplina frente a la obesidad. La invalidación de la experiencia subjetiva asociada a los procesos de alimentación se manifiesta en la falta de sensibilidad que expresan las madres para detectar el hambre y la saciedad de sus hijos(as). Esto influye en conductas de la madre de obligar al hijo a comer cuando éste no lo desea, lo que a su vez influye en la expresión de rechazo que manifiestan los niños para comer lo que les entrega la madre y que suele ser la comida sana. Otra influencia del desacoplamiento del estado mental del hijo(a) se expresa en la negación de la obesidad en el hijo, la cual lleva al descuido: la madre desconoce o confunde el peso de su hijo, no controla horarios, cantidades, ni tipos de comidas, deja que otros consientan al hijo con comida de forma ilimitada. Tanto el descuido de la madre frente a la alimentación como el rechazo de las comidas sanas del hijo influyen en que el niño(a) acceda a comer alimentos de alto contenido calórico y en forma excesiva, lo que influye físicamente en que el niño sea obeso(a).

Figura 1.

Diagrama codificación axial

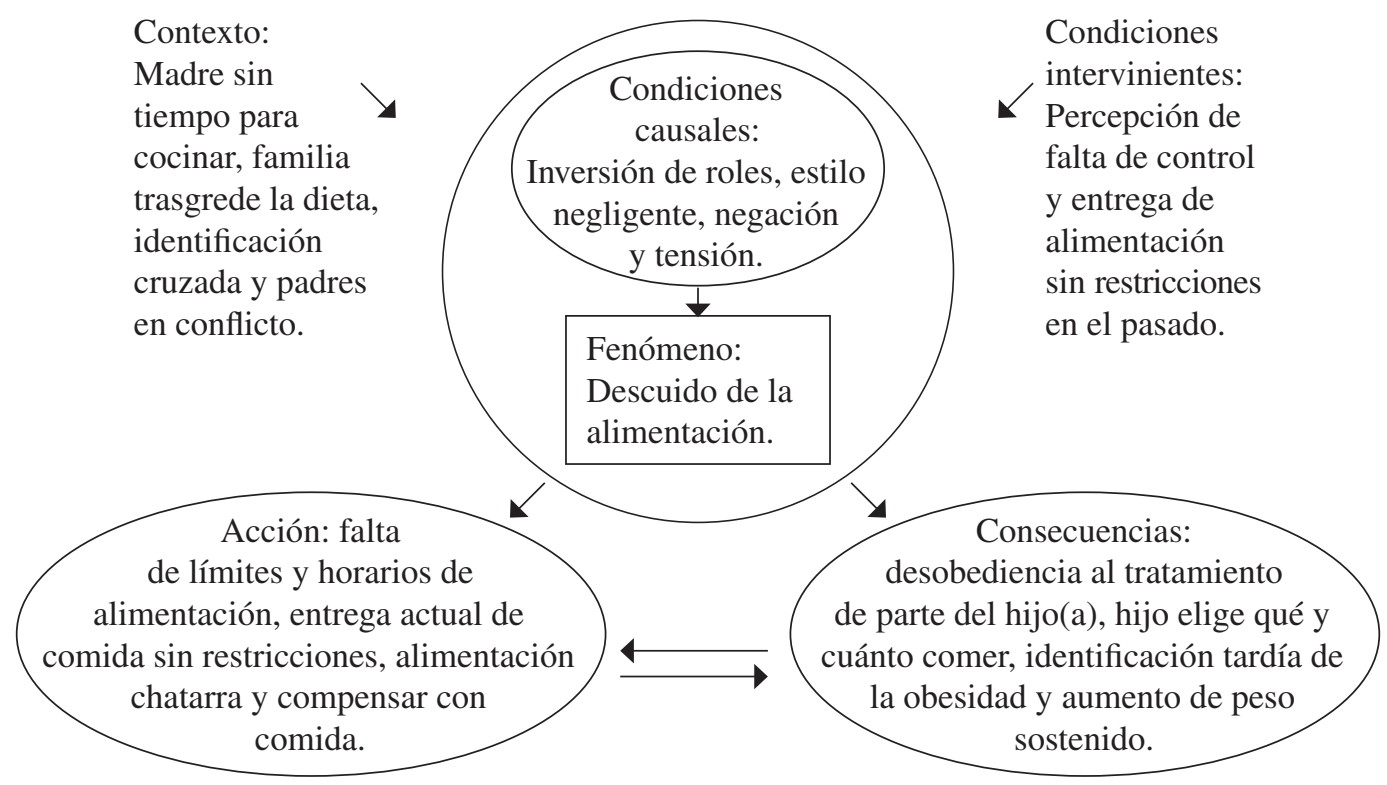

\footnotetext{
5 Se muestra el fenómeno del descuido de la alimentación y sus relaciones.
} 


\section{Discusión y conclusiones}

A partir del estudio es posible concluir que los niños con obesidad que participaron en la investigación presentan características de un apego inseguro con la madre, y que la respuesta sensible de sus madres está alterada y por lo tanto, la percepción de conductas alimenticias ligadas a la saciedad también lo está, generando un terreno proclive a la obesidad. A partir de los resultados es posible pensar que el hijo obeso compensa las necesidades emocionales de la madre, por lo que ella niega el problema y se tensiona en su solución. Las respuestas de la madre como el descuido, la invalidación de la experiencia subjetiva, la inversión de roles y la falta de sensibilidad para detectar el hambre y la saciedad del hijo, implicadas en el apego inseguro, se relacionan con la obesidad ya que favorecen el rechazo del hijo a la comida entregada por la madre y la ingesta excesiva de comida no saludable; por otro lado, estas respuestas maternas dificultan en el niño la autorregulación de la comida y la autopercepción de sus estados corporales.

En cuanto a las representaciones de apego de los(as) niños(as), es posible concluir que presentan un apego inseguro con sus madres. Esto es concordante con los estudios con la concepción de Guidano sobre los apegos inseguros y los trastornos de alimentación (1987, 1994). Por otro lado, la baja resolución de conflicto encontrada en todos los niños, se relaciona con estudios que han encontrado bajas habilidades sociales en pacientes obesos y abre posibilidades de intervención que favorezcan el aprendizaje de conductas asertivas frente a los conflictos y a la experimentación de la ira (Hidalgo y Glotieb, 2001).

En cuanto a las dificultades en la respuesta materna, el descuido en la alimentación presenta la relación más conductual entre respuesta materna y conductas en torno a la alimentación que favorecen la obesidad; a partir de este estudio, se comprende por una cadena de frustración en el rol materno - déficit en la respuesta sensible (Barudy y Dantagnan, 2005; Fonagy, et. al. 1998), que se expresa en severos problemas de control sobre la conducta de alimentación del hijo, dejando que éste coma en exceso comidas de alto contenido calórico. Los resultados del descuido muestran que también se expresa a nivel emocional, en el cual la madre falla en la contención emocional al hijo, prioriza sus propios estados emocionales por sobre los del hijo y le cuesta prestar su mentalismo en situaciones de estrés y en otras situaciones donde el niño la demanda, como en el caso del juego.
A la base de la frustración de la madre y por ende del descuido, se encuentran los significados de las necesidades emocionales que la madre proyecta satisfacer con su hijo, específicamente como el que llena su vacío. Esto es apoyado por la identificación del hijo como proyección de las fantasías de nutrición de su madre (Bustos, Fantuzzi, Greibe y Steiner, 2000), así como por la tendencia autoconfirmatoria y la respuesta disminuida a los cuidados del hijo frente a la frustración de las madres con apego inseguro-ambivalente (Barudy y Dantagnan, 2000). La proyección de necesidades emocionales en el hijo se observó en la negación del problema de obesidad del hijo por parte de la madre, lo que influye en que la identificación de la patología se realice de modo tardío, así como la constante tensión en la solución del problema ejecutando pautas ambivalentes y contradictorias. Lo anteriormente señalado debe ser incorporado en el tratamiento de la obesidad como un objetivo terapéutico, que facilite una conciencia del problema y que aborde los problemas de la madre para ayudar a seguir el tratamiento; esto es especialmente relevante en la negación, el descuido, la tensión o ambivalencia y la ansiedad frente a la ingesta del hijo. Esta es una área de trabajo para la prevención y tratamiento de la obesidad que involucra a las madres y que tendría por objetivo lograr una mayor conciencia sobre los significados en las relaciones con el hijo, de modo de ordenar y filtrar aquellas demandas al hijo que perjudiquen su autorregulación y autonomía (Bowlby, 1998; Crittenden, 1992). Por otro lado, la respuesta materna negligente de negación o traslado de responsabilidad, puede ser prevenida para apoyar la disminución de la cronificación y gravedad de la obesidad (Ainsworth, 1981).

A diferencia de lo que piensan las madres, no aparecen en el estudio datos fidedignos que avalen la existencia de obesidad debido a factores genéticos o a alguna otra patología de tipo física (Carmena, 1985, citado en Belloch, 1995); por otro lado, un número de los casos estudiados presentaron bajo peso al nacer, lo que contradice la idea de que el obeso nace gordo, pero que favorece la reacción de ansiedad de la madre para que el niño engorde. En cuanto a la baja actividad física asociada a la obesidad, se observó que la sobreprotección y el déficit en la sensibilidad de la madre limitan el movimiento del hijo. Se piensa que las modificaciones al comportamiento sedentario de los niños con obesidad deben incluir un trabajo con la sobreprotección y el déficit en la sensibilidad de la madre.

El intento de las madres por moderar la ingesta del hijo, no logra modificar las pautas de sobreprotección, 
el descuido y la invalidación de la experiencia subjetiva del hijo, especialmente en torno al hambre y saciedad del hijo. Esto nos muestra que cambios a la conducta por sí sola que no involucren un análisis más profundo de la relación madre-hijo tienden al fracaso (Bowlby, 1998), lo que puede observarse cuando se intenta un cambio en el obeso sólo en torno a la comida, estilo de tratamiento que ha presentado bajos resultados (Barja et al., 2001).

En cuanto a la invalidación de la experiencia subjetiva, el hijo rechaza a la madre cuando ella no lo ve en su subjetividad o cuando insiste con la comida que el hijo no quiere, no aceptando los alimentos que ella le ofrece. En respuesta a esta frustración, la madre actúa negligentemente dejando que el hijo coma lo que quiera y en exceso. Esta relación refiere características de un apego ansioso ambivalente. La posibilidad de intervención estaría enfocada en dotar de repertorio conductual adaptativo a la madre, cuando se produzca la parálisis de respuesta frente a la frustración y previamente cuando no logra respetar la autonomía del hijo (Barudy y Dantagnan, 2005). Estas intervenciones deben apuntar a aumentar la autoconciencia de los significados emocionales del hijo para la madre, la autoconfianza en su rol, a dotar de estrategias para manejar el estrés y para atender a las necesidades del hijo incorporando respuestas sensibles, dar mayor estructuración al niño y aprender a preparar adecuadamente el contexto (Crittenden, 1988).

En cuanto al desacoplamiento de las madres y las dificultades en la percepción de hambre y saciedad en el hijo, ellas tienden a entregar alimentos sin saber previamente si su hijo siente hambre; insisten en ofrecerles comidas incluso cuando el hijo intenta hacer dieta. Estos hechos nos muestran a una madre ansiosa y contradictoria, lo que favorece la inseguridad del apego y la ambivalencia (Bustos, et. al., 2000; Guidano, 1987). Se piensa que la madre produce una experiencia discrepante para el niño, no logrando poner límites ni organizar la experiencia en torno a la alimentación, pues vulnera la propia sensibilidad del niño en torno al hambre y la saciedad. Lo anterior no sólo se corresponde con un apego inseguro-ambivalente en el hijo, sino que refiere la dificultad de la madre de dotar de herramientas de organización que hagan suficientemente coherente la experiencia interna de hambre y saciedad del hijo. Estos resultados son concordantes con la descripción de los desórdenes alimentarios psicógenos (Guidano, 1987) y con otras evidencias del déficit en la percepción del hambre, el peso y las señales físicas de los niños obesos y de sus madres (Bustos, et. al., 2000; Díaz,
2000; Kain et al., 2001). Lo anterior hace pensar en el traspaso que hace la madre de esta incapacidad al hijo; las consecuencias de esta dificultad en la percepción estarían asociadas a la génesis y mantenimiento de la obesidad, que responde a hábitos de alimentación de inicio en la infancia (Fernández, 1994).

Las categorías encontradas en este estudio requieren otras investigaciones que contribuyan a reforzarlas y a aumentar su consistencia; el nivel observacional del apego necesita ser medido directamente en etapas tempranas de la infancia. Las dificultades en la respuesta materna sensible que tienen relación con la obesidad necesitan ser medidas en otros casos para aumentar la transferencia de estos resultados a más niños con obesidad.

Las proyecciones a nivel de investigación sugieren formular proyectos financiados que incorporen el diagnóstico y la intervención psicológica para la obesidad infantil, desde la respuesta materna sensible, el apego y la autopercepción y autorregulación de los estados internos del niño, de modo de determinar su efecto en el cambio de hábitos de alimentación, en la baja de peso y en la posibilidad de prevención del trastorno en etapas tempranas de la infancia.

\section{Referencias}

Ainsworth, M., Blehar, M., Waters, E. \& Wall, S. (1978). Patterns of Attachment. A Psychological Study of the Strange Situation. New Jersey: Lawrence Erlbaum Associates, Publishers.

Ainsworth, M. \& Tracy, R. (1981). Maternal affectionate behavior and infant-mother attachment patterns. Child Development. 52, 1341-1343.

Barja, S., Núñez, E., Ibarra, M., Velandia, S, Urrejola, P. \& Hodgson, M. (2001). "Efectividad a corto plazo del tratamiento de obesidad infantil". Departamentos de Pediatría y de Nutrición-DiabetesMetabolismo, PUC. Manuscrito no publicado. Programa de Magíster en Nutrición.

Barudy, J. \& Dantagnan, M. (2005). Los buenos tratos a la infancia. Parentalidad, apego y resiliencia. Barcelona: Gedisa.

Belloch, A., Sandin, B. \& Ramos, F. (1995): Manual de Psicopatología. Vol II. España: McGraw Hill.

Bowlby, J.(1997). El vínculo afectivo. Barcelona: Paidós.

Bowlby, J. (1998). El apego y la pérdida. Barcelona: Paidós.

Briones, G. (1982). Métodos y técnicas avanzadas de investigación aplicados a la educación y ciencias sociales. Santiago, Curso de Educación a Distancia.

Buceta, J. \& Bueno, A. (1996). Tratamiento psicológico de hábitos y enfermedades. Madrid: Pirámide.

Bustos, P., Fantuzzi, F. Greibe, C. y Steiner, V. (2000). Buscando la forma de trabajar con grupos de niños obesos. Trabajo presentado en el IX Congreso de la Asociación Mexicana de Psicoterapia Analítica de Grupo México.

Bustos, P., Fantuzzi, F., Greibe, C. \& Steiner, V. (s.f.). "Sobre la obesidad". 
Carretero, M., Marchesi, A., Palacios, J. (1983): "Psicología Evolutiva". Madrid: Alianza.

Colegio de Psicólogos de Chile (1999). Código de ética profesional. Santiago.

Crittenden, P. (1988). Family and dyadic patterns of functioning in maltreating families. En K. Browne, C. Davis and P. Stratton (Eds.). Early Prediction and prevention of child abuse (pp. 161189). London: John Wiley \& Sons Ltd.

Crittenden, P. (1995). Attachment at risk for psychopathology: the early years. Developmental and Behavioral Pediatrics, 16(3), 12-16.

Díaz, M. (2000): Percepción materna del estado nutritivo de sus hijos obesos. Revista Chilena de Pediatría, 71(4), 316-320.

Fernández - Ballesteros, R. (1994). Evaluación conductual hoy. Un enfoque para el cambio en psicología clínica y de la salud. Madrid: Pirámide.

Flick, U. (2002). An Introduction to Qualitative Research. London: Sage.

Fonagy, P., Target, M., Steele, H. y Steele, M. (1998). Reflective function manual for application to adult attachment interviews. London: University College London.

González, L., Hidalgo, M., Hurtado, M., Nova, C. \& Venegas, M. (2002). Relación entre factores individuales y familiares de riesgo para desórdenes alimenticios en alumnos de enseñanza media. Revista de Psicología de la Universidad de Chile, 11(1).

Guba, E. (1990). The paradigm dialog. Newbury Park: Sage.

Guidano, V. (1987). Complexity of the self. New York: The Guilford Press.

Guidano, V. (1994). El sí mismo en proceso. Buenos Aires: Paidós.

Hidalgo, C. \& Gottlieb, B. (2001). Comportamiento interpersonal de pacientes obesos y su relación con el éxito en un tratamiento para bajar de peso. PSYKHE, 10(1), 59-68.

Kain, J., Olivares, S., Castillo, M.y Vio, F. (2001). Validación y aplicación de instrumentos para evaluar Intervenciones educativas en obesidad de escolares. Revista Chilena de Pediatría, 72(4); 308-318.

Knapp, M. (1991). La comunicación no verbal: el cuerpo y el entorno. México: Paidós.
Krause, M. (1992). Métodos de la investigación cualitativa. Documento.

Krause, M. (1995). La investigación cualitativa: un campo de posibilidades y desafíos. Revista Temas de Educación, 7, 19-40.

Lecannelier, F. (2002). La entrevista de apego de niños Child Attachment Interview - CAI. Revista Terapia Psicológica, 20(1), 37, 53-60.

Main, M., Kaplan N. \& Cassidy, J. (1985). Security in infancy, childhood and adulthood: a move to the level of representation. Monographs of the Society for Research in Child Development; 50, 1-12.

Marrone, M. (2002). La teoría del apego. Un enfoque actual. Madrid: Psimática.

Martorell, M. \& González, R. (1997). Entrevista y consejo psicológico. Madrid: Síntesis psicológica.

Miller, W. (1980). The addictive behaviors. New York: Pergamon Press.

Olivares, C., Zacarías, H. \& Lera, M. (2005). Estado nutricional y consumo de alimentos seleccionados en escolares de la Región Metropolitana: Línea base para un proyecto de promoción del consumo de pescado. Revista chilena de nutrición. 32(2), 102-108.

Pérez Serrano, G. (1994). Investigación Cualitativa. Retos e Interrogantes. I - II . Madrid: La Muralla.

Plozza, L \& Pöldinger, W. (1986). El enfermo psicosomático, su médico práctico. Suiza: Roche.

Raich, R., Torras, J. \& Mora, M. (1997). Análisis estructural de variables influyentes en la aparición de alteraciones de la conducta alimentaria. Psicología Conductual. 5(1), 55-70.

Sroufe, L. (2000). Desarrollo emocional. La organización de la vida emocional en los primeros años. Oxford: University Press.

Taylor, S. \& Bogdan, R. (1992). Introducción a los métodos cualitativos de investigación. Barcelona: Paidós.

Target, M., Fonagy, P. \& Shmueli-Goetz, Y. (2003): Attachment representations in school-age children: development of the child attachment interview (CAI). Journal of Child Psychotherapy, 29(2), 171-186. 
